\title{
Study on Infinite Buffer Batch Size Dependent Bulk Service Queue with Queue Length Dependent Vacation
}

\author{
G. K. Tamrakar ${ }^{1}$. A. Banerjee ${ }^{1}$
}

Accepted: 29 October 2021 / Published online: 22 November 2021

(c) The Author(s), under exclusive licence to Springer Nature India Private Limited 2021

\begin{abstract}
Queueing models with vacations have drawn the attention of researchers over several decades as a handy tool for tackling real-life congestion problems. Keeping this in mind, we pay attention to an infinite buffer single server batch-size dependent batch service queue with queue size (queue length) dependent vacation. The arrival pattern of the customers in the system follows the Poisson process where they get the service in packets/group following the general batch service (GBS) rule. An embedded Markov chain technique is used for the mathematical analysis where service (vacation) completion epochs have been taken as an embedded Markov point. We obtain the bivariate generating functions of the queue size and vacation type (queue size at vacation initiation epoch) at vacation termination epoch, and the bivariate generating function of the queue size and batch size with the server at service completion epoch, and then we successfully extract the steady-state joint probabilities of the queue size and batch size with the server and the joint probabilities of the queue size and vacation type at various epochs. Finally, various performance measures are presented. Also, the behavior of the considered model is presented by the graphs and tables.
\end{abstract}

Keywords Non Poisson queue · Infinite buffer · Single (Multiple) vacation · Bivariate generating function

\section{Introduction}

Theory of the batch service queue with vacation has huge application in various congestion situations, viz., telecommunication, manufacturing, transportation, etc. In telecommunication system messages, data, digital signals are first broken into cells (packets) and are transmitted over the common transmission line in batches with a minimum threshold and maximum capacity and whenever the minimum number of packets is not available for the transmission, the multiplexer enters into the predefined single vacation or multiple vacation. In the batch

G. K. Tamrakar

gagankrtamrakar.rs.mat17@itbhu.ac.in

A. Banerjee

anuradha.mat@iitbhu.ac.in

1 Indian Institute of Technology (BHU), Varanasi, India 
service queue, a single server operates the customers in the groups/batches with various service rules, viz., general batch service (GBS) rule, arbitrary batch size service rule, fixed batch size service rule, etc. Neuts [22] proposed the GBS rule which is the most popular batch service rule in queueing literature in which the customers get serves in packets with lower threshold $a(\geq 1)$ and upper threshold $b$. For the specific analysis of batch service queue, we mention Chaudhry and Templeton [5] and Medhi [21], and for the current status on the literature of batch service queue, readers are invoked to see [1-3,10,24] and the references therein. In the batch service queue, the idle period of the server may be utilized in a customized manner by entangling the server in some secondary job. This period of absence of the server from the primary job facility is called the server vacation period.

In several queueing systems when no jobs (i.e., primary job) are available for the server, then the server performs some secondary jobs, this type of model is interpreted as the vacation queueing model, and proposed by Levy and Yechiali [20]. Later many researchers ([9], [32], [11], [15], [16] and the references therein) have been attracted to the queueing models with vacation phenomena, and in this connection, an impressive survey is presented in the survey papers by Doshi [8] and Ke et al. [17]. For the quality literature on vacation theory, readers are requested to see Takagi [29] and Tian and Zhang [31]. In vacation queue after one service, if sufficient number of customer is available in the queue, then the server continues the service, otherwise, it takes the vacation, and after the completion of vacation if the system contains sufficient number of job to start a service, then it starts a service, otherwise, remains in the dormant state ( single vacation, i.e., SV) or goes for another vacation (multiple vacation, i.e., MV).

Batch service queues with SV where the behavior of the system analyzed by the supplementary variable technique (SVT), can be found in $[12,13,19,26]$, in which authors have obtained queue size distribution at various epochs. batch service queue with MV applies in many real-life queues, viz., telecommunication, transportation, manufacturing, etc. In this connection, many queue theorists have been attracted to analyze such models, Choi and Han [6], Jeyakumar and Senthilnathan [14], etc. The batch service queues with SV as well as MV analyzed by a few researchers, Lee et al. [18], Samanta et al. [25], Sikdar and Gupta [27], Sikdar and Samanta [28], Gupta et al. [11], Tamrakar and Banerjee [30], and the references therein.

Most of the above literature on batch service queue with vacations deals with the derivation of the distribution of the queue size at various epochs only, in which the service time is considered to be independent of the batch size with the server. Though, Gupta et al. [11] considered $M / G_{r}^{(a, b)} / 1$ finite buffer queue with SV (MV). They obtained the required joint distributions at various epoch. By using the embedded Markov chain technique, they first derived transition probability matrix (TPM) to obtain the joint distribution of the queue size and batch size with the server at the epoch of service completion and the queue size and vacation type at vacation termination epoch. However, it seems difficult to handle TPM with a considerably large buffer size or an infinite buffer queue (see e.g., Bar-Lev et al. [4]). To address this difficulty, in this paper we present an analytical study of the batch service queue with SV (MV) and infinite buffer. where the service time and vacation time depend on the batch size with the server and queue size at the vacation initiation epoch, respectively. We first use SVT and obtain the bivariate generating function for the joint probabilities of the queue size and batch size with the server at service completion epoch and the queue size and vacation type at vacation termination epoch. Then we successfully extract all the required service (vacation) completion epoch joint probabilities which are eventually used to obtain corresponding arbitrary epoch joint probabilities. The analytical study presented in this paper 
is completely different than that is presented in Gupta et al. [11] and our result cannot be obtained as a special case of their result. The novelty of our work is that where the queueing practitioners stuck with the results provided by Gupta et al. [11] while handling an infinite buffer system, our results will definitely help them to find out the proper solution.

The practical motivation for the considered model comes up with the way of the sample testing procedure in the pandemic situation (viz., COVID-19). An effective way of fighting some dangerous virus (pandemic) such as the corona virus is to test all suspected people in whom the virus is likely to be found. However, at the beginning of an epidemic, the shortage of test kits causes major problems which aggravate the pandemic. To deal with such a situation group testing may play a key role instead of an individual test. In a group test, samples of multiple swabs are mixed to form a 'mixed sample', which is then tested. If the test result is found to be negative, then all samples in the mixture are negative for the virus, however, a positive test shows one or more samples infected with the virus in the mixture. Then that particular sample will be further tested to identify the infected samples. Such group testing process is already justified during the COVID-19 pandemic, see [7,23,33].

Our model may be helpful in policy-making for group testing methods to deal with a pandemic situation for a particular country. For better understanding let us consider an example of group testing in which the samples are coming according to the Poisson stream and the health worker test the samples in bulk (i.e., mixed sample) with a lower threshold ' $a$ ' to upper threshold ' $b$ ' (following GBS rule). On completion of a test if the number of waiting samples to be tested is $r(\geq a)$ then a mixed sample of $\operatorname{size} \min (r, b)$ is taken for the test. The mixing time depends on the number of samples which is going to be tested, therefore, batch size dependent service is justified. On the other hand, if at the completion of the test the number of waiting samples to be tested is $k(<a)$ then the health worker stops testing and goes on vacation. During vacation, the health worker will be engaged in some additional works (stocking of health care inventory, increase people's awareness, visiting the quarantine room, etc.). Before going for the vacation, the health worker always checks the queue size, and depending on the queue size he set his vacation time which will increase the expected number of samples tested per unit time. Hence, queue size dependent vacation is also justified.

The rest of the article is devoted as follows: Description of the considered model presented in Sect. 2. The steady-state joint probabilities is investigated at various epoch in Sect. 3. Significant marginal probabilities are presented in Sect. 4. Some necessary performance measures are reported in Sect. 5. Numerical results and their discussion are presented in Sect. 6. In Sect. 7, we develop a cost model in order to obtain the optimum value of the lower threshold $a$. The conclusion section ends the article.

\section{Model Description}

The present article investigates infinite capacity single server batch service queue with SV (MV) where the service time $\left(T_{r}\right)(1 \leq a \leq r \leq b)$ and vacation time $\left(V_{k}\right)(0 \leq k \leq a-1)$, respectively, depend on batch size with the server and queue size at vacation initiation epoch, respectively. The customers reach one by one in the system following the Poison distribution with a rate of $\lambda$, and are received the service in batches with a lower threshold $a(\geq 1)$ and upper threshold $b(b \geq a)$ as per the GBS rule. After service, if the queue size is $\geq a$ then the server renders the service as per the GBS rule otherwise the server goes for the vacation. The vacation time of the server depends on the size of the queue $k(0 \leq k \leq a-1)$ at the vacation initiation epoch. Such a vacation that depends on the queue size $k$ is known as $k^{t h}$ 
vacation type. There will be no permission to join any customer in the running service even if the server is serving the customers less than its maximum capacity. The service time $\left(T_{r}\right)$ $(1 \leq a \leq r \leq b)$ and the vacation time $\left(V_{k}\right)(0 \leq k \leq a-1)$ are distributed generally. Some other assumptions are as follows:

- $s_{r}(t)=$ probability density function (pdf) of $T_{r}$,

- $S_{r}(t)=$ distribution function (DF) of $T_{r}$,

- $\tilde{S}_{r}(\theta)=$ Laplace-Stieltjes transform (LST) of $T_{r}$,

- Mean service time $=\frac{1}{\mu_{r}}=s_{r}=-\tilde{S}_{r}^{(1)}(0)=$ derivative of $\tilde{S}_{r}(\theta)$ evaluated at $\theta=0$,

- $v_{k}(t)=$ pdf of $V_{k}$,

- $V_{k}(t)=$ DF of $V_{k}$,

- $\tilde{V}_{k}(\theta)=$ LST of $V_{k}$,

- Mean vacation time $=\frac{1}{v_{k}}=x_{k}=-\tilde{V}_{k}^{(1)}(0)=$ derivative of $\tilde{V}_{k}(\theta)$ evaluated at $\theta=0$.

After a $k^{t h}(0 \leq k \leq a-1)$ vacation type completion if the queue size $\geq a$, then the server renders the service as per the GBS rule, otherwise, following SV rule, the server remains in the dormant state until the queue size reaches the lower threshold $a$, or under MV rule, takes repeated vacation until it finds queue length $\geq a$ at the end of the vacation. The condition that ensures the system stability is $\frac{\lambda s_{b}}{b}(<1)$. In this article, we study both SV and MV queues simultaneously. By substituting $\delta=1$ in the steady-state analysis, we obtain the results for MV and by substituting $\delta=0$ we obtain the results for SV.

\section{System Analysis}

This section covers the analysis of the joint probabilities of the queue size and batch size with the server at the service completion epoch, and the joint probabilities of queue size and vacation type taken by the server at the vacation termination epoch. Then arbitrary epoch joint probabilities are obtained by establishing the relation between the joint probabilities of service (vacation) completion epoch and random epoch. For the mathematical analysis, using probability law, we get the steady-state equations by defining the additional variable for remaining service (vacation) time. To this end, the system state at time $t$ is introduced by random variables as follows:

- $N_{q}(t)$ represents the queue size.

- $N_{s}(t)$ represents the batch size with the server when the server is busy.

- $C(t)$ represents the vacation type taken by the server, when the server is on vacation.

- $X(t)$ represents remaining service time of the batch in service, if any.

- $Y(t)$ represents remaining vacation time of a vacation period, if any.

Note that the dormant state of the server at time $t$ will be represented by $N_{s}(t)=0$.

For SV, $\left\{\left(N_{q}(t), N_{s}(t)\right)\right\} \cup\left\{\left(N_{q}(t), N_{s}(t), X(t)\right) \cup\left(N_{q}(t), C(t), Y(t)\right)\right\}$ forms a Markov process with state space $\{(n, 0) ; 0 \leq n \leq a-1\} \bigcup\{(n, r, u) ; n \geq 0, a \leq r \leq b, u \geq$ $0\} \bigcup\{(n, k, u) ; 0 \leq k \leq a-1, n \geq k, u \geq 0\}$.

For MV, $\left\{\left(N_{q}(t), N_{s}(t), X(t)\right) \cup\left(N_{q}(t), C(t), Y(t)\right)\right\}$ forms a Markov process with state space $\{(n, r, u) ; n \geq 0, a \leq r \leq b, u \geq 0\} \bigcup\{(n, k, u) ; 0 \leq k \leq a-1, n \geq k, u \geq 0\}$.

Further, we set out the state probabilities at time $t$ as:

- $f_{n}(t) \equiv \operatorname{Pr}\left\{N_{q}(t)=n, N_{s}(t)=0\right\}, 0 \leq n \leq a-1$ (exist only for SV).

- $\alpha_{n, r}(u, t) d u \equiv \operatorname{Pr}\left\{N_{q}(t)=n, N_{s}(t)=r, u \leq X(t) \leq u+d u\right\}, n \geq 0, a \leq r \leq b$.

- $\beta_{n}^{[k]}(u, t) d u \equiv \operatorname{Pr}\left\{N_{q}(t)=n, C(t)=k, u \leq Y(t) \leq u+d u\right\}, \quad n \geq k, 0 \leq k \leq$ $a-1$. 
In steady state, as $t \rightarrow \infty$, we have,

$f_{n}=\lim _{t \rightarrow \infty} f_{n}(t)(0 \leq n \leq a-1)$ (exist only for SV),

$\alpha_{n, r}(u)=\lim _{t \rightarrow \infty} \alpha_{n, r}(u, t), \quad n \geq 0, \quad a \leq r \leq b$,

$\beta_{n}^{[k]}(u)=\lim _{t \rightarrow \infty} \beta_{n}^{[k]}(u, t), \quad n \geq k, \quad 0 \leq k \leq a-1$.

Now we obtain the system equation that governs the system behavior. Analyzing the model at time $t$ and $t+d t$, the governing equations in steady-state are as follows:

$$
\begin{aligned}
& 0=(1-\delta)\left(-\lambda f_{0}+\beta_{0}^{[0]}(0)\right), \\
& 0=(1-\delta)\left(-\lambda f_{n}+\lambda f_{n-1}+\sum_{k=0}^{n} \beta_{n}^{[k]}(0)\right) \text {, } \\
& 1 \leq n \leq a-1 \text {, } \\
& -\frac{d}{d u} \alpha_{0, a}(u)=-\lambda \alpha_{0, a}(u)+(1-\delta) \lambda f_{a-1} s_{a}(u) \\
& +\left(\sum_{k=0}^{a-1} \beta_{a}^{[k]}(0)+\sum_{r=a}^{b} \alpha_{a, r}(0)\right) s_{a}(u), \\
& -\frac{d}{d u} \alpha_{0, r}(u)=-\lambda \alpha_{0, r}(u)+\left(\sum_{k=0}^{a-1} \beta_{r}^{[k]}(0)+\sum_{j=a}^{b} \alpha_{r, j}(0)\right) s_{r}(u), \\
& a+1 \leq r \leq b \\
& -\frac{d}{d u} \alpha_{n, r}(u)=-\lambda \alpha_{n, r}(u)+\lambda \alpha_{n-1, r}(u), \quad a \leq r \leq b-1, \quad n \geq 1, \\
& -\frac{d}{d u} \alpha_{n, b}(u)=-\lambda \alpha_{n, b}(u)+\lambda \alpha_{n-1, b}(u) \\
& +\left(\sum_{k=0}^{a-1} \beta_{n+b}^{[k]}(0)+\sum_{r=a}^{b} \alpha_{n+b, r}(0)\right) s_{b}(u), n \geq 1, \\
& -\frac{d}{d u} \beta_{k}^{[k]}(u)=-\lambda \beta_{k}^{[k]}(u)+\left(\sum_{r=a}^{b} \alpha_{k, r}(0)+\delta \sum_{j=0}^{k} \beta_{k}^{[j]}(0)\right) v_{k}(u), \\
& 0 \leq k \leq a-1 \text {, } \\
& -\frac{d}{d u} \beta_{n}^{[k]}(u)=-\lambda \beta_{n}^{[k]}(u)+\lambda \beta_{n-1}^{[k]}(u), \quad n \geq k+1, \quad 0 \leq k \leq a-1 .
\end{aligned}
$$

Further, we define for $\operatorname{Re} \theta \geq 0$,

$$
\begin{aligned}
& \tilde{S}_{r}(\theta)=\int_{0}^{\infty} e^{-\theta u} d S_{r}(u)=\int_{0}^{\infty} e^{-\theta u} s_{r}(u) d u, \quad a \leq r \leq b, \\
& \tilde{\alpha}_{n, r}(\theta)=\int_{0}^{\infty} e^{-\theta u} \alpha_{n, r}(u) d u, \quad a \leq r \leq b, n \geq 0, \\
& \alpha_{n, r} \equiv \tilde{\alpha}_{n, r}(0)=\int_{0}^{\infty} \alpha_{n, r}(u) d u, \quad a \leq r \leq b, n \geq 0, \\
& \tilde{V}_{k}(\theta)=\int_{0}^{\infty} e^{-\theta u} d V_{k}(u)=\int_{0}^{\infty} e^{-\theta u} v_{k}(u) d u, \quad 0 \leq k \leq a-1, \\
& \tilde{\beta}_{n}^{[k]}(\theta)=\int_{0}^{\infty} e^{-\theta u} \beta_{n}^{[k]}(u) d u, \quad 0 \leq k \leq a-1, n \geq k,
\end{aligned}
$$




$$
\beta_{n}^{[k]} \equiv \tilde{\beta}_{n}^{[k]}(0)=\int_{0}^{\infty} \beta_{n}^{[k]}(u) d u, \quad 0 \leq k \leq a-1, \quad n \geq k
$$

One may note here that $\left(f_{n}\right)\left\{\alpha_{n, r}\right\}\left[\beta_{n}^{[k]}\right]$ denotes the probability of (queue size is $n$ and the sever is dormant, $0 \leq n \leq a-1)$ \{queue size is $n$ and batch size with server is $r, a \leq r \leq b$, $n \geq 0\}$ [queue size is $n$ and the server is on $k^{t h}$ vacation type, $0 \leq k \leq a-1, n \geq k$ ] at arbitrary epoch.

Our main objective is to obtain $f_{n}, \alpha_{n, r}$ and $\beta_{n}^{[k]}$ using (1)-(8). Keeping this in mind we multiply (3)-(8) by $e^{-\theta u}$ and integrate with respect to $u$ over the limits 0 to $\infty$, we get,

$$
\begin{aligned}
(\lambda-\theta) \tilde{\alpha}_{0, a}(\theta)= & (1-\delta) \lambda f_{a-1} \tilde{S}_{a}(\theta)+\left(\sum_{k=0}^{a-1} \beta_{a}^{[k]}(0)+\sum_{r=a}^{b} \alpha_{a, r}(0)\right) \tilde{S}_{a}(\theta) \\
& -\alpha_{0, a}(0), \\
(\lambda-\theta) \tilde{\alpha}_{0, r}(\theta)= & \left(\sum_{k=0}^{a-1} \beta_{r}^{[k]}(0)+\sum_{j=a}^{b} \alpha_{r, j}(0)\right) \tilde{S}_{r}(\theta) \\
& -\alpha_{0, r}(0), \quad a+1 \leq r \leq b, \\
(\lambda-\theta) \tilde{\alpha}_{n, r}(\theta)= & \lambda \tilde{\alpha}_{n-1, r}(\theta)-\alpha_{n, r}(0), n \geq 1, a \leq r \leq b-1, \\
(\lambda-\theta) \tilde{\alpha}_{n, b}(\theta)= & \lambda \tilde{\alpha}_{n-1, b}(\theta)+\left(\sum_{k=0}^{a-1} \beta_{n+b}^{[k]}(0)+\sum_{r=a}^{b} \alpha_{n+b, r}(0)\right) \tilde{S}_{b}(\theta) \\
& -\alpha_{n, b}(0), n \geq 1, \\
& -\sum_{k}^{b}(0), \quad 0 \leq k \leq a-1, \\
(\lambda-\theta) \tilde{\beta}_{k}^{[k]}(\theta)= & \left.\sum_{r=a}^{[k]} \alpha_{k, r}(0)+\delta \sum_{j=0}^{k} \beta_{k}^{[j]}(0)\right) \tilde{V}_{k}(\theta) \\
& \lambda \tilde{\beta}_{n-1}^{[k]}(\theta)-\beta_{n}^{[k]}(0) n \geq k+1, \quad 0 \leq k \leq a-1 .
\end{aligned}
$$

As our primary aim is to find the joint probabilities of the queue size as well as the batch size with the server (queue size and the vacation type of server) at an arbitrary epoch, which seems to be difficult to obtain directly from (15)-(20). Hence, we characterize the system state at service (vacation) completion epoch which reduces the continuous time Markov process into an embedded Markov chain where embedded Markov points are defined as service completion epoch and vacation termination epoch. The approach of finding an embedded Markov chain reduces the complexity of the system for mathematical evaluation. Towards this end, we set out the following probabilities at service (vacation) completion epoch as follows:

$$
\begin{aligned}
\alpha_{n, r}^{+}= & \operatorname{Pr}\{\text { queue size is } n \text { at service completion epoch of a batch size } r\}, n \geq 0, a \leq r \leq b, \\
\alpha_{n}^{+}= & \operatorname{Pr}\{\text { queue size is } n \text { at service completion epoch of a batch }\} \\
= & \sum_{r=a}^{b} \alpha_{n, r}^{+}, \quad n \geq 0, \\
\beta_{n}^{[k]+}= & \operatorname{Pr}\left\{\text { queue size is } n \text { at } k^{\text {th }}\right. \text { vacation type } \\
& \quad \text { termination epoch\}, } 0 \leq k \leq a-1, n \geq k, \\
\beta_{n}^{+}= & \operatorname{Pr} \text { \{queue size is } n \text { at the vacation termination epoch\} }
\end{aligned}
$$




$$
=\sum_{k=0}^{\min (n, a-1)} \beta_{n}^{[k]+}, \quad n \geq 0 .
$$

\section{Joint Probabilities at Service (Vacation) Completion Epoch}

In this section, our objective is to find $\alpha_{n, r}^{+}\left(a \leq r \leq b, n \geq 0\right.$, ) and $\beta_{n}^{[k]+}(0 \leq k \leq$ $a-1, n \geq k)$, In this connection we define few generating functions are as follows:

$$
\begin{aligned}
P(z, y, \theta) & =\sum_{n=0}^{\infty} \sum_{r=a}^{b} \tilde{\alpha}_{n, r}(\theta) z^{n} y^{r},|z| \leq 1,|y| \leq 1, \\
P^{+}(z, y)= & \sum_{n=0}^{\infty} \sum_{r=a}^{b} \alpha_{n, r}^{+} z^{n} y^{r},|z| \leq 1,|y| \leq 1, \\
P^{+}(z)= & \sum_{n=0}^{\infty} \sum_{r=a}^{b} \alpha_{n, r}^{+} z^{n}=\sum_{n=0}^{\infty} \alpha_{n}^{+} z^{n},|z| \leq 1, \\
Q(z, y, \theta)= & \sum_{k=0}^{a-1} \sum_{n=k}^{\infty} \tilde{\beta}_{n}^{[k]}(\theta) z^{n} y^{k},|z| \leq 1,|y| \leq 1, \\
Q^{+}(z, y)= & \sum_{k=0}^{a-1} \sum_{n=k}^{\infty} \beta_{n}^{[k]+} z^{n} y^{k},|z| \leq 1,|y| \leq 1, \\
Q^{+}(z)= & \sum_{k=0}^{a-1} \sum_{n=k}^{\infty} \beta_{n}^{[k]+} z^{n}=\sum_{n=0}^{\infty} \sum_{k=0}^{\min (n, a-1)} \beta_{n}^{[k]+} z^{n} \\
= & \sum_{n=0}^{\infty} \beta_{n}^{+} z^{n},|z| \leq 1 .
\end{aligned}
$$

Further, we set out the following probabilities

$$
\begin{aligned}
m_{j}^{(r)} & =\operatorname{Pr}\{j \text { customers arrive during the service of the batch size } r\}, j \geq 0, a \leq r \leq b, \\
& =\int_{0}^{\infty} \frac{e^{-\lambda t}(\lambda t)^{j}}{j !} s_{r}(t) d t, \\
w_{j}^{(k)} & =\operatorname{Pr}\left\{j \text { customers arrive during the } k^{t h} \text { vacation type }\right\}, j \geq 0,0 \leq k \leq a-1, \\
& =\int_{0}^{\infty} \frac{e^{-\lambda t}(\lambda t)^{j}}{j !} v_{k}(t) d t,
\end{aligned}
$$

such that $\sum_{j=0}^{\infty} m_{j}^{(r)}=1, \sum_{j=0}^{\infty} w_{j}^{(k)}=1$.

The PGF (probability generating function) of $m_{j}^{(r)}$ and $w_{j}^{(k)}$ are defined as follows:

$$
\begin{aligned}
& M^{(r)}(z)=\sum_{j=0}^{\infty} m_{j}^{(r)} z^{j}=\tilde{S}_{r}(\lambda-\lambda z), \quad a \leq r \leq b, \quad|z| \leq 1, \\
& N^{(k)}(z)=\sum_{j=0}^{\infty} w_{j}^{(k)} z^{j}=\tilde{V}_{k}(\lambda-\lambda z), \quad 0 \leq k \leq a-1, \quad|z| \leq 1 .
\end{aligned}
$$


Lemma 1 The joint probabilities $\alpha_{n, r}^{+}, \beta_{n}^{[k]+}, \alpha_{n, r}(0)$ and $\beta_{n}^{[k]}(0)(a \leq r \leq b, 0 \leq k \leq a-1)$ are associated with the following relation

$$
\begin{aligned}
\alpha_{n, r}^{+} & =\sigma \alpha_{n, r}(0), \quad n \geq 0, \\
\beta_{n}^{[k]+} & =\sigma \beta_{n}^{[k]}(0), \quad n \geq k,
\end{aligned}
$$

where $\sigma^{-1}=\sum_{m=0}^{\infty} \sum_{r=a}^{b} \alpha_{m, r}(0)+\sum_{m=0}^{\infty} \sum_{k=0}^{\min (m, a-1)} \beta_{m}^{[k]}(0)$.

Proof Since $\alpha_{n, r}^{+}$and $\beta_{n}^{[k]+}$ are proportional to $\alpha_{n, r}(0)$ and $\beta_{n}^{[k]}(0)$, respectively, using the concept of Bayes' theorem and $\sum_{n=0}^{\infty} \sum_{r=a}^{b} \alpha_{n, r}^{+}+\sum_{n=0}^{\infty} \sum_{k=0}^{\min (n, a-1)} \beta_{n}^{[k]+}=1$ we get the desired outcome.

Lemma 2 The value $\sigma^{-1}$ is given by

$$
\sigma^{-1}=\frac{1-(1-\delta) \sum_{n=0}^{a-1} f_{n}}{s_{b} \sum_{n=b+1}^{\infty}\left(\alpha_{n}^{+}+\beta_{n}^{+}\right)+\sum_{n=a}^{b}\left(\alpha_{n}^{+}+\beta_{n}^{+}\right) s_{n}+\sum_{n=0}^{a-1}\left(\alpha_{n}^{+} x_{n}+(1-\delta) \beta_{n}^{+} s_{a}+\delta \beta_{n}^{+} x_{n}\right)} .
$$

Proof Using (1) and (2), we obtain

$$
\lambda f_{n}=\sum_{m=0}^{n} \sum_{k=0}^{m} \beta_{m}^{[k]}(0), \quad 0 \leq n \leq a-1 .
$$

Using (37) in (15), we get

$$
(\lambda-\theta) \tilde{\alpha}_{0, a}(\theta)=\sum_{n=0}^{a-1} \sum_{k=0}^{n} \beta_{n}^{[k]}(0) \tilde{S}_{a}(\theta)+\left(\sum_{k=0}^{a-1} \beta_{a}^{[k]}(0)+\sum_{r=a}^{b} \alpha_{a, r}(0)\right) \tilde{S}_{a}(\theta)-\alpha_{0, a}(0) .
$$

Summing (38) and (16)-(20), we get

$$
\begin{aligned}
\sum_{m=0}^{\infty} \sum_{r=a}^{b} \alpha_{m, r}(\theta)+\sum_{m=0}^{\infty} \sum_{k=0}^{m i n(m, a-1)} \beta_{m}^{[k]}(\theta)= & \frac{1-\tilde{S}_{b}(\theta)}{\theta} \sum_{n=b+1}^{\infty}\left(\sum_{r=a}^{b} \alpha_{n, r}(0)+\sum_{k=0}^{a-1} \beta_{n}^{[k]}(0)\right) \\
& +\sum_{n=a}^{b}\left(\sum_{r=a}^{b} \alpha_{n, r}(0)+\sum_{k=0}^{a-1} \beta_{n}^{[k]}(0)\right) \frac{1-\tilde{S}_{n}(\theta)}{\theta} \\
& +\sum_{n=0}^{a-1}\left(\sum_{r=a}^{b} \alpha_{n, r}(0)+\delta \sum_{k=0}^{n} \beta_{n}^{[k]}(0)\right) \frac{1-\tilde{V}_{n}(\theta)}{\theta} \\
& +(1-\delta) \frac{1-\tilde{S}_{a}(\theta)}{\theta} \sum_{n=0}^{a-1} \sum_{k=0}^{n} \beta_{n}^{[k]}(0) .
\end{aligned}
$$

Taking $\theta \rightarrow 0$ in (39) and using L'Hôspital's rule, the normalization condition (1 б) $\sum_{n=0}^{a-1} f_{n}+\sum_{n=0}^{\infty} \sum_{r=a}^{b} \alpha_{n, r}+\sum_{n=0}^{\infty} \sum_{k=0}^{\min (n, a-1)} \beta_{n}^{[k]}=1$, after few algebraic calculation we get the desired outcome.

\section{Lemma 3}

$$
Q^{+}(z)=\sum_{k=0}^{a-1} \sum_{n=k}^{\infty} \beta_{n}^{[k]+} z^{n}=\sum_{k=0}^{a-1}\left(\alpha_{k}^{+}+\delta \beta_{k}^{+}\right) N^{(k)}(z) z^{k}
$$


Proof Multiplying (19) and (20) by the appropriate power of $z$ and $y$, and adding them over the range of $n$ and $k$, we get

$$
\begin{aligned}
& (\lambda-\theta-\lambda z) Q(z, y, \theta)=\sum_{k=0}^{a-1}\left(\sum_{r=a}^{b} \alpha_{k, r}(0)+\delta \sum_{j=0}^{k} \beta_{j}^{[k]}(0)\right) \tilde{V}_{k}(\theta) z^{k} y^{k} \\
& -\sum_{k=0}^{a-1} \sum_{n=k}^{\infty} \beta_{n}^{[k]}(0) z^{n} y^{k} .
\end{aligned}
$$

Now substituting $\theta=\lambda-\lambda z$ in (41) and using Lemma 1, (21) and (23) we obtain

$$
\sum_{k=0}^{a-1} \sum_{n=k}^{\infty} \beta_{n}^{[k]+} z^{n} y^{k}=\sum_{k=0}^{a-1}\left(\alpha_{k}^{+}+\delta \beta_{k}^{+}\right) N^{(k)}(z) z^{k} y^{k} .
$$

Substituting $y=1$ in (42) we acquire the desired outcome.

\section{Lemma 4}

$$
\beta_{n}^{[k]+}=\left(\alpha_{k}^{+}+\delta \sum_{j=0}^{k} \beta_{k}^{[j]+}\right) w_{n-k}^{(k)}, \quad 0 \leq k \leq a-1, n \geq k
$$

Proof From (42) collecting the coefficients of $y^{k}(0 \leq k \leq a-1)$ we obtain,

$$
\sum_{n=k}^{\infty} \beta_{n}^{[k]+} z^{n}=\left(\alpha_{k}^{+}+\delta \beta_{k}^{+}\right) N^{(k)}(z) z^{k} .
$$

Now using (33) and (23), in (44) and collecting the coefficients of $z^{n}(n \geq k$ ) we obtain desired result (43).

Hence from Lemma 4 it is clear that once $\alpha_{k}^{+}(0 \leq k \leq a-1)$ are known, the joint probabilities $\beta_{n}^{[k]+}(0 \leq k \leq a-1, n \geq k)$ are all known.

Now we turn our focus is to find the bivariate generating function for the queue size and batch size with the server at service completion epoch. Towards this end, multiplying (15)-(18) by appropriate power of $z$ and $y$ and adding them over the range of $n$ and $r$ we obtain,

$$
\begin{aligned}
(\lambda-\theta-\lambda z) P(z, y, \theta)= & (1-\delta) \sum_{n=0}^{a-1} \sum_{k=0}^{n} \beta_{n}^{[k]}(0) \tilde{S}_{a}(\theta) y^{a} \\
& +\sum_{r=a}^{b}\left(\sum_{k=0}^{a-1} \beta_{r}^{[k]}(0)+\sum_{j=a}^{b} \alpha_{r, j}(0)\right) \tilde{S}_{r}(\theta) y^{r} \\
& +\sum_{n=b+1}^{\infty}\left(\sum_{k=0}^{a-1} \beta_{n}^{[k]}(0)+\sum_{r=a}^{b} \alpha_{n, r}(0)\right) \tilde{S}_{b}(\theta) z^{n-b} y^{b} \\
& -\sum_{n=0}^{\infty} \sum_{r=a}^{b} \alpha_{n, r}(0) z^{n} y^{r} .
\end{aligned}
$$


Substituting $\theta=(\lambda-\lambda z)$ in the above expression and using Lemma 1, (21), (23) and (25), we get

$$
\begin{aligned}
P^{+}(z, y)= & (1-\delta) y^{a} M^{(a)}(z) \sum_{n=0}^{a-1} \beta_{n}^{+}+\sum_{r=a}^{b}\left(\beta_{r}^{+}+\alpha_{r}^{+}\right) M^{(r)}(z) y^{r} \\
& +\sum_{n=b+1}^{\infty}\left(\beta_{n}^{+}+\alpha_{n}^{+}\right) M^{(b)}(z) z^{n-b} y^{b} .
\end{aligned}
$$

substituting $y=1$ in (46) and using Lemma 3 and (26), we get the following result,

$$
P^{+}(z)=\frac{\left\{\begin{array}{c}
\sum_{n=0}^{a-1}\left[M^{(b)}(z)\left(\alpha_{n}^{+}+\delta \beta_{n}^{+}\right)\left(N^{(n)}(z)-1\right) z^{n}+(1-\delta) \beta_{n}^{+}\left(M^{(a)}(z) z^{b}-M^{(b)}(z) z^{n}\right)\right] \\
\left.+\sum_{n=a}^{b-1}\left(\beta_{n}^{+}+\alpha_{n}^{+}\right)\left(M^{(n)}(z) z^{b}-M^{(b)}(z) z^{n}\right)\right\}
\end{array}\right.}{z^{b}-M^{(b)}(z)} .
$$

Finally, using (47) in (46) after some algebraic manipulation we obtain,

$$
P^{+}(z, y)=\frac{\begin{array}{c}
\sum_{n=0}^{a-1}\left[(1-\delta) \beta_{n}^{+}\left(z^{b} y^{a} M^{(a)}(z)-y^{b} M^{(b)}(z) z^{n}\right)+(1-\delta) M^{(a)}(z) M^{(b)}(z)\left(y^{b}-y^{a}\right) \beta_{n}^{+}\right. \\
\left.+y^{b}\left(\alpha_{n}^{+}+\delta \beta_{n}^{+}\right)\left(N^{(n)}(z)-1\right) M^{(b)}(z) z^{n}\right] \\
+\sum_{n=a}^{b-1}\left(\beta_{n}^{+}+\alpha_{n}^{+}\right)\left(z^{b} y^{n} M^{(n)}(z)+\left(y^{b}-y^{n}\right) M^{(n)}(z) M^{(b)}(z)-y^{b} M^{(b)}(z) z^{n}\right)
\end{array}}{z^{b}-M^{(b)}(z)} .
$$

Remark 1 The bivariate generation function (44) of the queue size and the vacation type at vacation termination epoch, and the bivariate generating function of the queue size and batch size with the server at service completion epoch, i.e., (48) have not analyzed in the literature so far.

It may be observed from (48) that the generating function $P^{+}(z, y)$ is in the compact form, excluding the the $b$ unknowns $\left\{\alpha_{n}^{+}\right\}_{n=0}^{b-1}$. One may further note from Lemma 4 that once $\alpha_{k}^{+}$ $(0 \leq k \leq a-1)$ are known then the joint probabilities $\beta_{n}^{[k]+}(0 \leq k \leq a-1)$ are completely known. Hence, in order to find $\alpha_{n, r}^{+}(a \leq r \leq b, n \geq 0)$ and $\beta_{n}^{[k]+}(0 \leq k \leq a-1, n \geq k)$, one should first find the unknowns $\left\{\alpha_{n}^{+}\right\}_{n=0}^{b-1}$. Next section is dedicated in getting these unknowns $\left\{\alpha_{n}^{+}\right\}_{n=0}^{b-1}$.

\section{Procedure of Getting the Unknowns $\alpha_{n}^{+}(0 \leq n \leq b-1)$}

Note that the unknowns $\alpha_{n}^{+}(0 \leq n \leq b-1)$, as appeared in (48), are the same as the unknowns which are appeared in (47). Using the Rouche's theorem one may conclude that, for $\rho<1, z^{b}-M^{(b)}(z)$ has $(b-1)$ zeros, say $\hat{e}_{1}, \hat{e}_{2}, \ldots, \hat{e}_{l}$, with multiplicity $r_{1}, r_{2}, \ldots, r_{l}$, respectively, inside the unit circle $|z|=1$ (where $(l \leq b-1)$ and $\sum_{i=1}^{l} r_{i}=(b-1)$ ) and one simple zero, say, $z_{b}=1$, on the unit circle $|z|=1$. Due to analyticity of (47) in $|z| \leq 1$ these zeros are also the zeros of numerator of (47). Hence, from (47) we have $(b-1)$ linearly independent equations,

$$
\left[\frac { d ^ { i - 1 } } { d z ^ { i - 1 } } \left(\sum_{n=0}^{a-1}\left\{M^{(b)}(z)\left(\alpha_{n}^{+}+\delta \beta_{n}^{+}\right)\left(N^{(n)}(z)-1\right) z^{n}+(1-\delta) \beta_{n}^{+}\left(M^{(a)}(z) z^{b}-M^{(b)}(z) z^{n}\right)\right\}\right.\right.
$$




$$
\left.\left.+\sum_{n=a}^{b-1}\left(\beta_{n}^{+}+\alpha_{n}^{+}\right)\left(M^{(n)}(z) z^{b}-M^{(b)}(z) z^{n}\right)\right)\right]_{z=\hat{e}_{j}}=0,1 \leq j \leq l \& 1 \leq i \leq r_{j},
$$

where $\frac{d^{0}}{d z^{0}} h(z) \equiv h(z)$.

Now using (47), Lemma 3 and the normalization condition $P^{+}(1)+Q^{+}(1)=1$, applying L'Hôspital's rule, we get

$$
\begin{aligned}
& \sum_{n=0}^{a-1}\left[\left(\alpha_{n}^{+}+\delta \beta_{n}^{+}\right)\left(\lambda x_{n}+b-b \rho\right)+(1-\delta) \beta_{n}^{+}(b-n)\right] \\
& \quad+\sum_{n=a}^{b-1}\left[\left(\beta_{n}^{+}+\alpha_{n}^{+}\right)\left(b-n+\lambda\left(s_{n}-s_{b}\right)\right)\right]=b-b \rho .
\end{aligned}
$$

Hence, (49) and (50) together forms non-homogenous system of $b$ linearly independent equations in $b$ unknowns $\alpha_{n}^{+}(0 \leq n \leq b-1)$, solving them we uniquely determine $\alpha_{n}^{+}$ $(0 \leq n \leq b-1)$.

Theorem 1 The joint probabilities $\alpha_{n, r}^{+}(1 \leq a \leq r \leq b-1, n \geq 0)$ are given by

$$
\begin{aligned}
& \alpha_{n, a}^{+}=\left((1-\delta) \sum_{m=0}^{a-1} \beta_{m}^{+}+\beta_{a}^{+}+\alpha_{a}^{+}\right) m_{n}^{(a)}, \\
& \alpha_{n, r}^{+}=\left(\beta_{r}^{+}+\alpha_{r}^{+}\right) m_{n}^{(r)}, a+1 \leq r \leq b-1 .
\end{aligned}
$$

Proof Using (25) in (48) and then accumulating the coefficients of $y^{r}(1 \leq a \leq r \leq b-1)$, we obtain

$$
\begin{aligned}
& \text { coefficient of } y^{a}: \sum_{n=0}^{\infty} \alpha_{n, a}^{+} z^{n}=\left((1-\delta) \sum_{m=0}^{a-1} \beta_{m}^{+}+\beta_{a}^{+}+\alpha_{a}^{+}\right) M^{(a)}(z) . \\
& \text { coefficient of } y^{r}: \sum_{n=0}^{\infty} \alpha_{n, r}^{+} z^{n}=\left(\beta_{r}^{+}+\alpha_{r}^{+}\right) M^{(r)}(z), a+1 \leq r \leq b-1 .
\end{aligned}
$$

Using (32) in (53) and (54), and then accumulating the coefficients of $z^{n}$, we readily obtain the desired result (51) and (52).

We now proceed with our mathematical analysis to achieve the remaining joint probabilities $\alpha_{n, b}^{+}(n \geq 0)$. To get these, we use (25) in (48), and then accumulating the coefficient of $y^{b}$, we obtain

$$
\begin{array}{r}
M^{(b)}(z)\left\{\sum_{n=0}^{a-1}\left[\left(\alpha_{n}^{+}+\delta \beta_{n}^{+}\right)\left(N^{(n)}(z)-1\right) z^{n}+(1-\delta) \beta_{n}^{+}\left(M^{(a)}(z)-z^{n}\right)\right]\right. \\
\sum_{n=0}^{\infty} \alpha_{n, b}^{+} z^{n}=\frac{\left.+\sum_{n=a}^{b-1}\left(\beta_{n}^{+}+\alpha_{n}^{+}\right)\left(M^{(n)}(z)-z^{n}\right)\right\}}{z^{b}-M^{(b)}(z)} .
\end{array}
$$

Now to derive $\alpha_{n, b}^{+}(n \geq 0)$ completely we need to invert the right hand side of (55) and towards this direction we consider LST of service time and the vacation time distribution as $\tilde{S}_{r}(\theta)=\frac{P_{r}(\theta)}{Q_{r}(\theta)}, a \leq r \leq b$, and $\tilde{V}_{k}(\theta)=\frac{P_{k}(\theta)}{Q_{k}(\theta)}, 0 \leq k \leq a-1$, respectively. Here one 
should note that the logic behind the consideration of $\tilde{S}_{r}(\theta)$ and $\tilde{V}_{k}(\theta)$ in rational form is that, in most of real life queueing model service (vacation) time distribution can be expressed as rational function. However, the transcendental LST (for example LST of deterministic distribution) can be handle using Padé approximation.

Now substituting $M^{(r)}(z)=\tilde{S}_{r}(\lambda-\lambda z)=\frac{P_{r}(\lambda-\lambda z)}{Q_{r}(\lambda-\lambda z)}, a \leq r \leq b$ and $N^{(k)}(z)=$ $\tilde{V}_{k}(\lambda-\lambda z)=\frac{P_{k}(\lambda-\lambda z)}{Q_{k}(\lambda-\lambda z)}, 0 \leq k \leq a-1$, in the right hand side of (55), after some simplification (55) can be converted as,

$$
\sum_{n=0}^{\infty} \alpha_{n, b}^{+} z^{n}=\frac{U(z)}{D(z)}
$$

where $U(z)$ and $D(z)$ are polynomials of degree $\bar{u}$ and $d$, respectively, and $D(z)$ is a monic polynomial (i.e., the coefficient of $z^{d}$ in $D(z)$ is 1 ). To extract the joint probabilities $\alpha_{n, b}^{+}$ $(n \geq 0)$ the zeros of $D(z)$ of modules greater than one must be known. Due to analyticity of (56), in $|z| \leq 1$, the zeros of $D(z)$ which lie inside and on the unit circle are also the zeros of $U(z)$, therefore, they can not play any role in extracting $\alpha_{n, b}^{+}(n \geq 0)$. Towards this end, let $\gamma_{1}, \gamma_{2}, \ldots, \gamma_{l}$ be the zeros of $D(z)$ of modules greater than one with multiplicity $\tau_{1}, \tau_{2}, \ldots, \tau_{l}$, respectively, such that $\sum_{j=1}^{l} \tau_{j} \leq d$. Now two cases may arise depending on $d$ and $\bar{u}$. We address these two cases as follows:

Case A: $d \leq \bar{u}$

Applying the method of partial fraction on (56), $\sum_{n=0}^{\infty} \alpha_{n, b}^{+} z^{n}$ is given by,

$$
\sum_{n=0}^{\infty} \alpha_{n, b}^{+} z^{n}=\sum_{i=0}^{\bar{u}-d} \varrho_{i} z^{i}+\sum_{j=1}^{l} \sum_{i=1}^{\tau_{j}} \frac{B_{i, j}}{\left(z-\gamma_{j}\right)^{\tau_{j}-i+1}}
$$

where

$$
B_{i, j}=\frac{1}{(i-1) !}\left[\frac{d^{i-1}}{d z^{i-1}}\left(\frac{U(z) \frac{d^{\tau_{j}}}{d z^{\tau_{j}}}\left(z-\gamma_{j}\right)^{\tau_{j}}}{\frac{d^{\tau_{j}}}{d z^{\tau_{j}}}(D(z))}\right)\right]_{z=\gamma_{j}}, j=1,2, \ldots, l, i=1,2, \ldots, \tau_{j} .
$$

Accumulating the coefficients of $z^{n}(n \geq 0)$ from (57) we get,

$$
\alpha_{n, b}^{+}= \begin{cases}\varrho_{n}+\sum_{j=1}^{l} \sum_{i=1}^{\tau_{j}} \frac{B_{i, j}}{(-1)^{\tau_{j}-i+1} \gamma_{j}^{\tau_{j}+n-i+1}}\left(\begin{array}{c}
\tau_{j}-i+n \\
\tau_{j}-i
\end{array}\right), & 0 \leq n \leq \bar{u}-d, \\
\sum_{j=1}^{l} \sum_{i=1}^{\tau_{j}} \frac{B_{i, j}}{(-1)^{\tau_{j}-i+1} \gamma_{j}^{\tau_{j}+n-i+1}}\left(\begin{array}{c}
\tau_{j}-i+n \\
\tau_{j}-i
\end{array}\right), & n>\bar{u}-d .\end{cases}
$$

Case $B: d>\bar{u}$

We eliminate first summation of the right-hand side of (57) and hence $\alpha_{n, b}^{+}$is given by,

$$
\alpha_{n, b}^{+}=\sum_{j=1}^{l} \sum_{i=1}^{\tau_{j}} \frac{B_{i, j}}{(-1)^{\tau_{j}-i+1} \gamma_{j}^{\tau_{j}+n-i+1}}\left(\begin{array}{c}
\tau_{j}-i+n \\
\tau_{j}-i
\end{array}\right), n \geq 0 .
$$

Thus, we have completed the analysis of the joint probabilities $\alpha_{n, r}^{+}(a \leq r \leq b, n \geq 0)$ at service completion epoch and $\beta_{n}^{[k]+}(0 \leq k \leq a-1, n \geq k)$ at the vacation termination epoch. Now we turn our objective for getting these probabilities at arbitrary epoch.

Remark 2 By inverting the PGF $M^{(r)}(z)$ and $N^{(k)}(z)$ we can easily compute $m_{j}^{(r)}, a \leq r \leq$ $b, j \geq 0$ and $w_{j}^{(k)}, 0 \leq k \leq a-1, j \geq 0$. 
Theorem 2 The probabilities $f_{n}(0 \leq n \leq a-1), \alpha_{n, r}(n \geq 0, a \leq r \leq b)$ and $\beta_{n}^{[k]}$ $(n \geq k, 0 \leq k \leq a-1)$ are given by,

$$
\begin{aligned}
f_{n} & =\frac{\sum_{m=0}^{n} \beta_{m}^{+}}{E}, 0 \leq n \leq a-1(\text { for } S V) \\
\alpha_{n, a} & =\frac{(1-\delta) \sum_{m=0}^{a-1} \beta_{m}^{+}+\beta_{a}^{+}-\sum_{j=0}^{n} \alpha_{j, a}^{+}+\alpha_{a}^{+}}{E}, n \geq 0, \\
\alpha_{n, r} & =\frac{\beta_{r}^{+}+\alpha_{r}^{+}-\sum_{j=0}^{n} \alpha_{j, r}^{+}}{E}, n \geq 0, a+1 \leq r \leq b-1, \\
\alpha_{n, b} & =\frac{\sum_{j=0}^{n}\left(\beta_{b+j}^{+}+\alpha_{b+j}^{+}-\alpha_{j, b}^{+}\right)}{E}, n \geq 0, \\
\beta_{n}^{[k]} & =\frac{\alpha_{k}^{+}+\delta \beta_{k}^{+}-\sum_{j=k}^{n} \beta_{j}^{[k]+}}{E}, n \geq k, 0 \leq k \leq a-1,
\end{aligned}
$$

where $E=\lambda g+(1-\delta) \sum_{i=0}^{a-1}(a-i) \beta_{i}^{+}$,

$g=s_{b} \sum_{n=b+1}^{\infty}\left(\alpha_{n}^{+}+\beta_{n}^{+}\right)+\sum_{n=a}^{b}\left(\alpha_{n}^{+}+\beta_{n}^{+}\right) s_{n}+\sum_{n=0}^{a-1}\left(\alpha_{n}^{+} x_{n}+(1-\delta) \beta_{n}^{+} s_{a}+\delta \beta_{n}^{+} x_{n}\right)$.

Proof Dividing (1) by $\sigma^{-1}$ and using Lemma 1, Lemma 2 and (23), we obtain

$$
f_{0}=\frac{\left(1-\sum_{n=0}^{a-1} f_{n}\right) \beta_{0}^{+}}{\lambda g} \text {. }
$$

Similarly, from (37), we obtain

$$
f_{n}=\frac{\left(1-\sum_{i=0}^{a-1} f_{i}\right) \sum_{m=0}^{n} \beta_{m}^{+}}{\lambda g}, 0 \leq n \leq a-1 .
$$

Using (65) in (66), we obtain

$$
f_{n}=\frac{f_{0}}{\beta_{0}^{+}} \sum_{m=0}^{n} \beta_{m}^{+}, \quad 0 \leq n \leq a-1 .
$$

Using (67) in (65) after some algebraic manipulation, we obtain

$$
f_{0}=\frac{\beta_{0}^{+}}{\lambda g+\sum_{i=0}^{a-1}(a-i) \beta_{i}^{+}} .
$$

Using (68) in (67), we obtain

$$
f_{n}=\frac{\sum_{m=0}^{n} \beta_{m}^{+}}{\lambda g+\sum_{i=0}^{a-1}(a-i) \beta_{i}^{+}}, 0 \leq n \leq a-1 .
$$

Setting $\theta=0$ in (15)-(20), we get

$$
\begin{aligned}
\lambda \alpha_{0, a} & =(1-\delta) \sum_{m=0}^{a-1} \sum_{k=0}^{m} \beta_{m}^{[k]}(0)+\sum_{k=0}^{a-1} \beta_{a}^{[k]}(0)+\sum_{r=a}^{b} \alpha_{a, r}(0)-\alpha_{0, a}(0) \\
\lambda \alpha_{0, r} & =\sum_{k=0}^{a-1} \beta_{r}^{[k]}(0)+\sum_{j=a}^{b} \alpha_{r, j}(0)-\alpha_{0, r}(0), a+1 \leq r \leq b
\end{aligned}
$$




$$
\begin{aligned}
\lambda \alpha_{n, r} & =\lambda \alpha_{n-1, r}-\alpha_{n, r}(0), n \geq 1, a \leq r \leq b-1, \\
\lambda \alpha_{n, b} & =\lambda \alpha_{n-1, b}+\sum_{k=0}^{a-1} \beta_{n+b}^{[k]}(0)+\sum_{r=a}^{b} \alpha_{n+b, r}(0)-\alpha_{n, b}(0), \quad n \geq 1, \\
\lambda \beta_{k}^{[k]} & =\sum_{r=a}^{b} \alpha_{k, r}(0)+\delta \sum_{j=0}^{k} \beta_{k}^{[j]}(0)-\beta_{k}^{[k]}(0), \quad 0 \leq k \leq a-1, \\
\lambda \beta_{n}^{[k]} & =\lambda \beta_{n-1}^{[k]}-\beta_{n}^{[k]}(0), n \geq k+1,0 \leq k \leq a-1 .
\end{aligned}
$$

Dividing (70) and (71) by $\sigma^{-1}$, respectively, and then using Lemma 1, Lemma 2, (21) and (23), we obtain

$$
\begin{aligned}
\alpha_{0, a} & =\frac{\left(1-(1-\delta) \sum_{i=0}^{a-1} f_{i}\right)\left((1-\delta) \sum_{m=0}^{a-1} \beta_{m}^{+}+\beta_{a}^{+}+\alpha_{a}^{+}-\alpha_{0, a}^{+}\right)}{\lambda g}, \\
\alpha_{0, r} & =\frac{\left(1-(1-\delta) \sum_{i=0}^{a-1} f_{i}\right)\left(\beta_{r}^{+}+\alpha_{r}^{+}-\alpha_{0, r}^{+}\right)}{\lambda g} .
\end{aligned}
$$

Using (65) and (68) in (76)-(77), respectively, we obtain

$$
\begin{aligned}
\alpha_{0, a} & =\frac{\left((1-\delta) \sum_{m=0}^{a-1} \beta_{m}^{+}+\beta_{a}^{+}+\alpha_{a}^{+}-\alpha_{0, a}^{+}\right)}{\lambda g+(1-\delta) \sum_{i=0}^{a-1}(a-i) \beta_{i}^{+}}, \\
\alpha_{0, r} & =\frac{\left(\beta_{r}^{+}+\alpha_{r}^{+}-\alpha_{0, r}^{+}\right)}{\lambda g+(1-\delta) \sum_{i=0}^{a-1}(a-i) \beta_{i}^{+}}, a+1 \leq r \leq b .
\end{aligned}
$$

Applying similar process for (72)-(73) and using (78) and (79), we obtain

$$
\begin{aligned}
\alpha_{n, a}= & \frac{\left((1-\delta) \sum_{m=0}^{a-1} \beta_{m}^{+}+\beta_{a}^{+}+\alpha_{a}^{+}-\sum_{j=0}^{n} \alpha_{j, a}^{+}\right)}{\lambda g+(1-\delta) \sum_{i=0}^{a-1}(a-i) \beta_{i}^{+}}, n \geq 1, \\
\alpha_{n, r}= & \frac{\left(\beta_{r}^{+}+\alpha_{r}^{+}-\sum_{j=0}^{n} \alpha_{j, r}^{+}\right)}{\lambda g+(1-\delta) \sum_{i=0}^{a-1}(a-i) \beta_{i}^{+}}, n \geq 1, a+1 \leq r \leq b-1, \\
\alpha_{n, b}= & \frac{\sum_{j=0}^{n}\left(\beta_{b+j}^{+}+\alpha_{b+j}^{+}-\alpha_{j, b}^{+}\right)}{\lambda g+(1-\delta) \sum_{i=0}^{a-1}(a-i) \beta_{i}^{+}}, n \geq 1 .
\end{aligned}
$$

Combining (78) and (80) we obtain (61). Combining (79) and (81) over the range $r$, we obtain (62) and (63).

Dividing (74) by $\sigma^{-1}$ and using Lemma 1, Lemma 2 and (21), we obtain

$$
\beta_{k}^{[k]}=\frac{\left(1-(1-\delta) \sum_{i=0}^{a-1} f_{i}\right)\left(\alpha_{k}^{+}+\delta \beta_{k}^{+}-\beta_{k}^{[k]+}\right)}{\lambda g}, 0 \leq k \leq a-1 .
$$

Using (66) and (68) in (83), we obtain

$$
\beta_{k}^{[k]}=\frac{\left(\alpha_{k}^{+}+\delta \beta_{k}^{+}-\beta_{k}^{[k]+}\right)}{\lambda g+(1-\delta) \sum_{i=0}^{a-1}(a-i) \beta_{i}^{+}}, \quad 0 \leq k \leq a-1 .
$$


Applying similar process for (75) after some algebraic manipulation, we obtain

$$
\beta_{n}^{[k]}=\frac{\left(\alpha_{k}^{+}+\delta \beta_{k}^{+}-\sum_{j=k}^{n} \beta_{j}^{[k]+}\right)}{\lambda g+(1-\delta) \sum_{i=0}^{a-1}(a-i) \beta_{i}^{+}}, n \geq k+1,0 \leq k \leq a-1 .
$$

Combining (84)-(85) we get (64). Now by back substitution method we simply obtain the joint probabilities $\beta_{n}^{[k]}(0 \leq k \leq a-1, n \geq k)$ from (64).

\section{Marginal Probabilities}

Marginal probabilities that can be found from the earlier results are as follows:

1. Queue size distribution is

$$
P_{n}^{\text {quеие }}=\left\{\begin{array}{l}
(1-\delta) f_{n}+\sum_{r=a}^{b} \alpha_{n, r}+\sum_{k=0}^{\min (n, a-1)} \beta_{n}^{[k]}, 0 \leq n \leq a-1, \\
\sum_{r=a}^{b} \alpha_{n, r}+\sum_{k=0}^{\min (n, a-1)} \beta_{n}^{[k]}, n \geq a .
\end{array}\right.
$$

2. Probability that the server is in a dormant state $\left(=P_{d o r}\right)=(1-\delta) \sum_{n=0}^{a-1} f_{n}$.

3. Probability of the batch size with the server is $r\left(=P_{r}^{\text {ser }}\right)=\sum_{n=0}^{\infty} \alpha_{n, r}, a \leq r \leq b$.

4. Probability that the server is in $k^{\text {th }}$ vacation type $\left(=Q_{v a c}^{[k]}\right)=\sum_{n=k}^{\infty} \beta_{n}^{[k]}, 0 \leq k \leq a-1$.

5. Probability that the server is busy $\left(=P_{\text {busy }}\right)=\sum_{r=a}^{b} \sum_{n=0}^{\infty} \alpha_{n, r}$.

6. Probability that the server is on vacation $\left(=Q_{v a c}\right)=\sum_{k=0}^{a-1} \sum_{n=k}^{\infty} \beta_{n}^{[k]}$.

7. Probability that the server is idle $\left(=P_{\text {idle }}\right)=(1-\delta) P_{d o r}+Q_{v a c}$.

\section{Some New Results as Particular Cases}

In this section, we present some useful results which seem to be new in the literature. These results derive from the results obtained for the considered queuing model as particular cases.

- The present paper analyzes the batch size-dependent service $M / G_{r}^{(a, b)} / 1$ queue and queue size dependent SV (MV). Hence, If we consider $\mu_{i}=\mu, a \leq i \leq b ; v_{i}=v$, $0 \leq i \leq a-1$ then the considered model is reduces to batch size independent service $M / G^{(a, b)} / 1$ queue with SV and MV where the vacation time is not dependent to queue size at vacation initiation epoch. This reduced model is analyzed by Sikdar and Gupta [26] for SV only, and they obtained only queue size distributions at various epoch. For verification if we substitute $\delta=0, M^{(r)}(z)=\tilde{M}(z),(a \leq r \leq b)$ and $N^{(k)}(z)=\tilde{N}(z)$ $(0 \leq k \leq a-1)$ in (47) and (40) we obtain the service completion epoch (vacation completion epoch) generating function for queue size distribution as follows,

$$
\begin{aligned}
& \tilde{M}(z)\left[\sum_{n=0}^{a-1}\left(\alpha_{n}^{+}(\tilde{N}(z)-1) z^{n}+\beta_{n}^{+}\left(z^{b}-z^{n}\right)\right)\right. \\
& P^{+}(z)=\frac{\left.+\sum_{n=a}^{b-1}\left(\beta_{n}^{+}+\alpha_{n}^{+}\right)\left(z^{b}-z^{n}\right)\right]}{z^{b}-\tilde{M}(z)}, \\
& Q^{+}(z)=\tilde{N}(z) \sum_{k=0}^{a-1} \alpha_{k}^{+} z^{k},
\end{aligned}
$$


which matches exactly with the results obtained by Sikdar and Gupta [26, eq(43), eq(44), page 953]. Further, from (42) and (48) we obtain

$$
\begin{aligned}
& Q^{+}(z, y)=\tilde{N}(z) \sum_{k=0}^{a-1}\left(\alpha_{k}^{+}+\delta \beta_{k}^{+}\right) z^{k} y^{k} \\
& \tilde{M}(z) \sum_{n=0}^{a-1}\left[(1-\delta) \beta_{n}^{+}\left(z^{b} y^{a}-y^{b} z^{n}\right)+(1-\delta) \tilde{M}(z)\left(y^{b}-y^{a}\right) \beta_{n}^{+}\right. \\
& \left.+y^{b}\left(\alpha_{n}^{+}+\delta \beta_{n}^{+}\right)(\tilde{N}(z)-1) z^{n}\right] \\
& P^{+}(z, y)=\frac{+\tilde{M}(z) \sum_{n=a}^{b-1}\left(\beta_{n}^{+}+\alpha_{n}^{+}\right)\left(z^{b} y^{n}+\left(y^{b}-y^{n}\right) \tilde{M}(z)-y^{b} z^{n}\right)}{z^{b}-\tilde{M}(z)} \text {, }
\end{aligned}
$$

which are bivariate generating functions at vacation termination epoch, and service completion epoch, respectively, and is not available so far in the literature. From these bivariate generating functions ((88) and (89)), applying similar procedure as presented in this paper, one can obtain the complete joint probabilities of the queue size and batch size with the server ; also, the joint probabilities of the queue size and vacation type at any time point.

- If we consider $a=1$ then the presented model converts in $M / G_{r}^{(1, b)} / 1$ queue with SV (MV). According to the analysis done in this paper, we can extract joint probabilities at different epochs. Such results are also new contributions to the literature.

- If we take $a=b$ then the present model reduces to $M / G^{b} / 1$ queue with queue size dependent SV and MV.

\section{Performance Measure}

The performance measure is the procedure that collects the information of the system and helps the manager to run the system smoothly. The present section covers significant performance measures of the considered model.

1. Expected queue size $\left(L_{q}\right)$ is given by

$$
\begin{aligned}
& L_{q}=(1-\delta) \sum_{n=0}^{a-1} n f_{n}+\sum_{n=0}^{\infty} \sum_{r=a}^{b} n \alpha_{n, r}+\sum_{k=0}^{a-1} \sum_{n=k}^{\infty} n \beta_{n}^{[k]}=(1-\delta) \\
& \sum_{n=0}^{a-1} n P_{n}^{\text {queue }}+\sum_{n=a-\delta a}^{\infty} n P_{n}^{\text {queue }} .
\end{aligned}
$$

2. Expected system size $\left(L_{S}\right)$ is given by

$$
L_{s}=(1-\delta) \sum_{n=0}^{a-1} n f_{n}+\sum_{n=0}^{\infty} \sum_{r=a}^{b}(n+r) \alpha_{n, r}+\sum_{k=0}^{a-1} \sum_{n=k}^{\infty} n \beta_{n}^{[k]} .
$$

3. Expected waiting time of a customer in the queue $\left(W_{q}\right)$ is given by $W_{q}=\frac{L_{q}}{\lambda}$.

4. Expected waiting time of a customer in the system $\left(W_{s}\right)$ is given by $W_{s}=\frac{L_{s}}{\lambda}$.

5. Expected batch size with the server when server is busy $\left(L^{\text {ser }}\right)$ is given by $L^{\text {ser }}=\sum_{r=a}^{b}\left(r P_{r}^{\text {ser }} / P_{\text {busy }}\right)$.

6. Expected vacation type taken by server when server is in vacation ( $L^{v a c}$ ) is given by $L^{v a c}=\sum_{k=0}^{a-1}\left(k Q_{v a c}^{[k]} / Q_{v a c}\right)$. 


\section{Numerical Results}

In this section, we present a variety of numerical results to show the behavior of the performance measures of the model under study, using graphs and tables. In this connection, we first consider the example of a sugarcane mill, which is presented by Tamrakar and Banerjee [30], however, with proper modifications as per our considered model. This example will reflect the more original scenario of the sugarcane mill example and also the real-life applicability of our considered model. Let us consider that the sugarcane machine takes 3 to 6 packets of sugarcane for producing juice. After a production if the machine finds 3 or more packets in the queue then it produces juice as per GBS rule, i.e., machine takes $l=\min (r, 6)$ packets for producing the juice with service rate $\mu_{l},\left(\mu_{3}=5.16666, \mu_{4}=3.87500, \mu_{5}=3.100000\right.$, and $\mu_{6}=2.583333$ ) otherwise, the machine performs either $0^{\text {th }}$ vacation type or $1^{\text {th }}$ vacation type or $2^{\text {th }}$ vacation type. The service (juice producing) time and the vacation time follow $E_{3}$ distribution and exponential distribution, respectively. In the $0^{\text {th }}$ vacation type, the machine removes waste, checks the machinery parts, and purifies the extracted juice assembled in the containers, However, in $1^{\text {th }}$ or $2^{\text {th }}$ vacation type, it checks the machinery parts. We assume that the packets are arriving with rate $\lambda=4.5$ following the Poisson manner. Then we observe the following results, where $v_{0}=1.3, v_{1}=1.7$, and $v_{2}=1.9$. (i.e., for the case of queue size dependent vacation (QSDV)).

Average packet (customer) size in the queue Average waiting time of a packet in the queue
2.817 (for the case of SV) 0.626 (for the case of SV)
3.373 (for the case of MV) 0.749 (for the case of MV)

We observe the following results, where $v_{0}=v_{1}=v_{2}=1.3$. (i.e., for the case of queue size independent vacation (QSIV)).

Average packet (customer) size in the queue Average waiting time of a packet in the queue
3.334 (for the case of SV)

0.740 (for the case of SV)
4.036 (for the case of MV) 0.897 (for the case of MV)

From the above findings we can conclude that, for this particular example, the average queue size (waiting time) for QSDV is less than the average queue size (waiting time) for QSIV. Hence, the consideration of QSDV makes the model more efficient than QSIV.

For further justification of our considered model graphically, we have introduced a comparison between QSDV and QSIV (see, Figs. 1, 2, 3, 4, 5, 6 and 7).

For the comparison purpose we consider the following two cases:

Case 1. The QSDV rates are taken as $v_{k}=(k+1)^{2} 0.85,0 \leq k \leq a-1$.

Case 2. The QSIV rates are taken as $v_{k}=v_{0}, 0 \leq k \leq a-1$. In case 1 , the vacation rates are chosen in such a way that as queue size at vacation initiation epoch increases the vacation time decreases accordingly. However, for case 2, the vacation time remains constant irrespective of the queue size at vacation initiation epoch. In Figs. 1, 2, 3, 4, 5, 6 and 7 we present the comparison between QSDV and QSIV for the $M / G_{r}^{(4,9)} / 1$ queue with SV (MV). Figures 1, 2, 3 and 4 represent the influence of the arrival rate $\lambda$ on $L_{q}$ and $W_{q}$. The service time follows Erlang $\left(E_{2}\right)$ distribution with batch size-dependent service rate $\mu_{r}=\frac{\mu}{r}, \quad 4 \leq r \leq 9$ 
Fig. 1 Effect of $\lambda$ on $L_{q}$

Fig. 2 Effect of $\lambda$ on $L_{q}$

Fig. 3 Effect of $\lambda$ on $W_{q}$
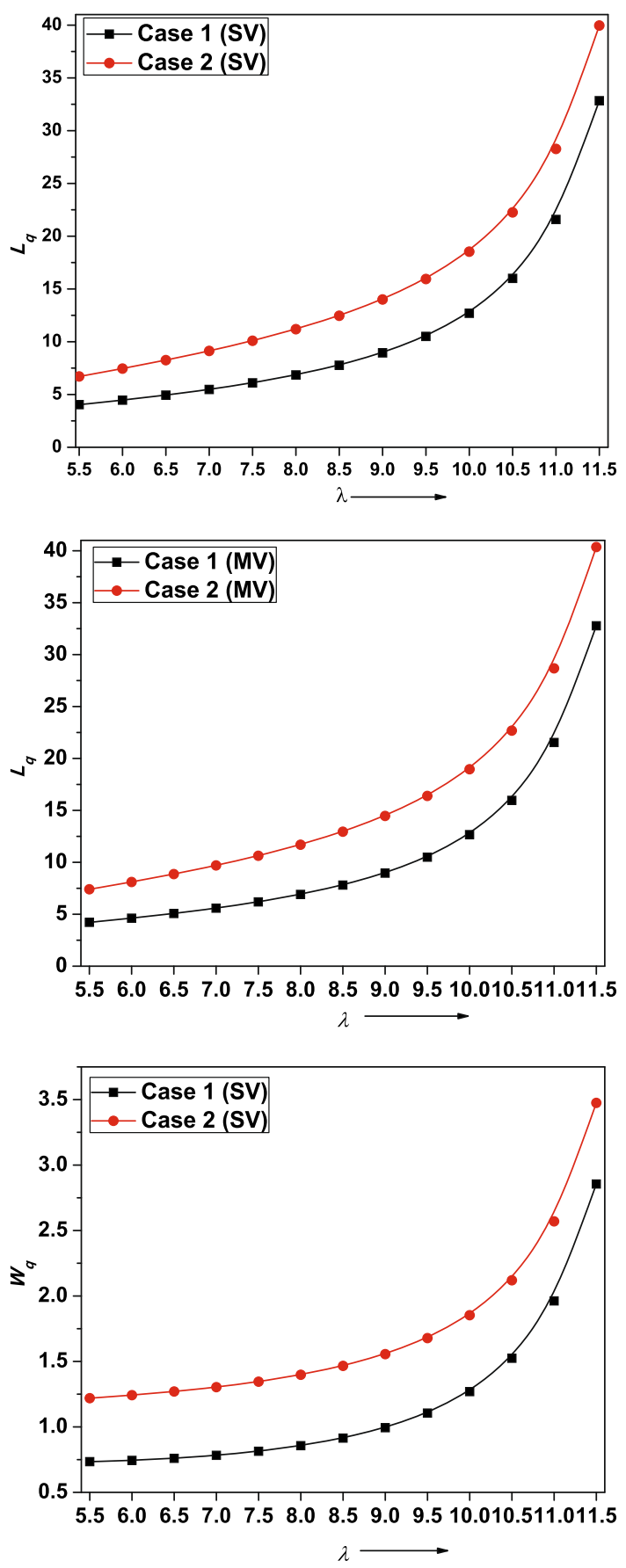
Fig. 4 Effect of $\lambda$ on $W_{q}$

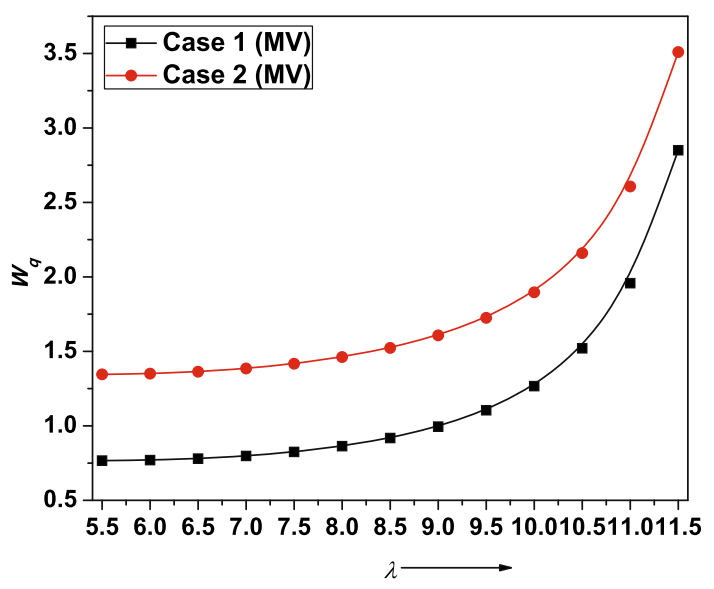

Fig. 5 Effect of $\lambda$ on $P_{d o r}$

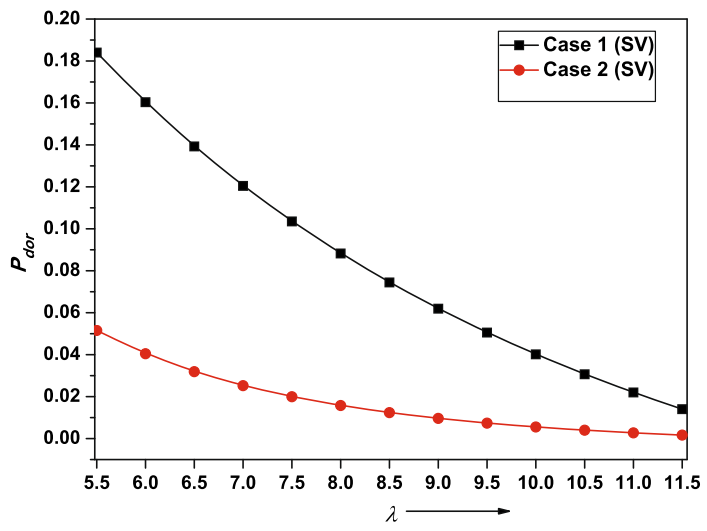

Fig. 6 Effect of $\lambda$ on $Q_{v a c}$

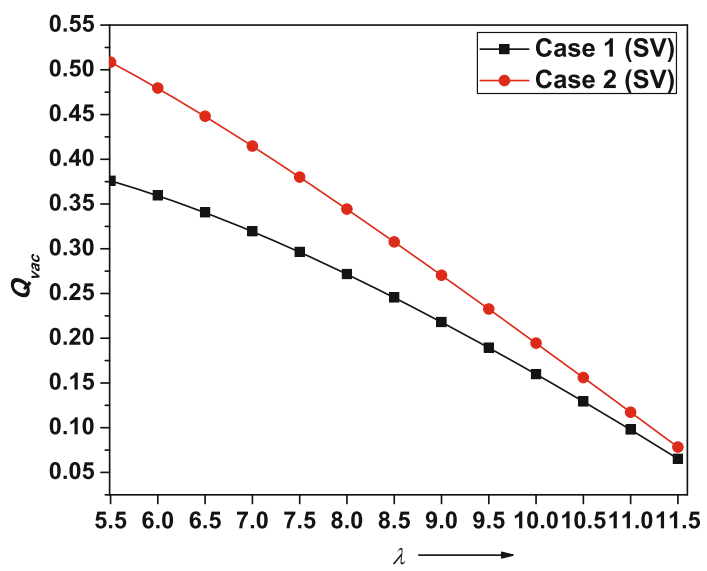


Fig. 7 Effect of $\lambda$ on $Q_{v a c}$

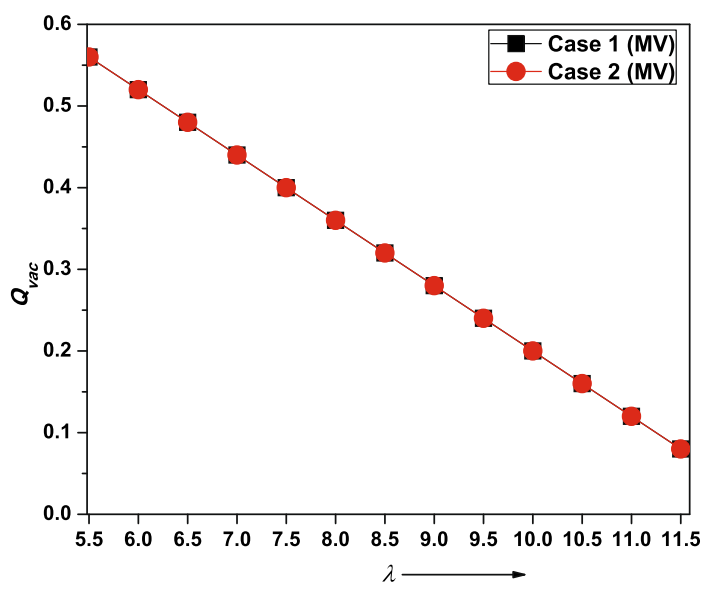

where $\mu=12.5$, and the vacation time distributed exponentially. The above consideration holds for both the cases, i.e., Case 1 and Case 2. It is observed from Figs. 1, 2, 3, 4 that as $\lambda$ increases from 5.5 to 11.5 (i.e., $\rho$ varies from 0.32 to 0.92 ) the performance measures $L_{q}$ and $W_{q}$ increases in both the cases. Also, it is noted that for a fixed $\lambda, L_{q}\left(W_{q}\right)$ is lower in Case 1 as compared to Case 2. Hence, the above studies reveals that assumption of QSDV policy in the batch size-dependent service model is more efficient, as QSDV is minimizing $L_{q}$ and $W_{q}$, in comparison to the QSIV policy.

In Fig. $5, P_{d o r}$ is depicted versus $\lambda$ for SV. It is observed that as $\lambda$ increases from 5.5 to 11.5, i.e., $\rho$ varies from 0.32 to $0.92, P_{\text {dor }}$ decreases for both the cases. This is because the mean vacation time of the server is longer for Case 2 than in Case 1, which means that the returning time (from vacation) of the server in the system from the vacation is shorter in Case 1 as compared to Case 2. Hence, the reflexion in Fig. 5 is on the expected direction in the sense that for a fixed value of $\lambda, P_{d o r}$ is greater for Case 1 in comparison to Case 2.

In Fisg. 6 and 7 we present the effect of $\lambda$ on $Q_{v a c}$ for SV and MV, respectively. It is observed that the increase in $\lambda$ from 5.5 to 11.5 , results in a decrease in $Q_{v a c}$ for both the cases, which is on the expected line.

In Fig. 8 we have plotted the effect of $\lambda$ versus $Q_{v a c}$ for SV (Case1) and MV (Case 1). The input parameters and the service (vacation) time distributions are taken exactly as we have taken for Figs. 1, 2, 3, 4, 5, 6 and 7. From Fig. 8 it is observed that as $\lambda$ increases from 5.5 to $11.5, Q_{v a c}$ decreases. Since increase in value of $\rho$ from 0.32 to 0.92 results in increase in $L_{q}$ significantly, i.e., the probability that the server is busy should also increase. The influence of $\lambda$ on $Q_{v a c}$ presented in Fig. 8 is on the expected direction, as for the case of SV, $Q_{v a c}=P_{\text {idle }}-P_{\text {dor }}$ and for the case of $\mathrm{MV} Q_{v a c}=P_{\text {idle }}$.

In Figs. 9 and 10 we present the effect of $\lambda$ on $L_{q}$ and $Q_{v a c}$, respectively, for $M / G_{r}^{(3,7)} / 1$ queue with SV for different vacation time distribution, e.g., (Exponential (M), Erlang $\left(E_{2}\right)$ and Deterministic (D)) with rate $v_{k}=(k+1)^{2} 0.95(0 \leq k \leq 2)$. Service time of each batch distributed exponentially with rate $\mu_{r}=\frac{\mu}{r}, 3 \leq r \leq 7$ where $\mu=7.5$, irrespective of vacation time distribution. From Figs. 9 and 10, as $\lambda$ increases from 3 to 7, i.e., $\rho$ increases from 0.4 to $0.93, L_{q}$ increases and $Q_{v a c}$ decreases. All the numerical experiments that are presented here in the form of graphs (Figs. 1, 2, 3, 4, 5, 6, 7, 8, 9 and 10) helps us to understand that whether our main objective of studying the proposed model is actually 
Fig. 8 Effect of $\lambda$ on $Q_{v a c}$

Fig. 9 Effect of $\lambda$ on $L_{q}$

Fig. 10 Effect of $\lambda$ on $Q_{v a c}$
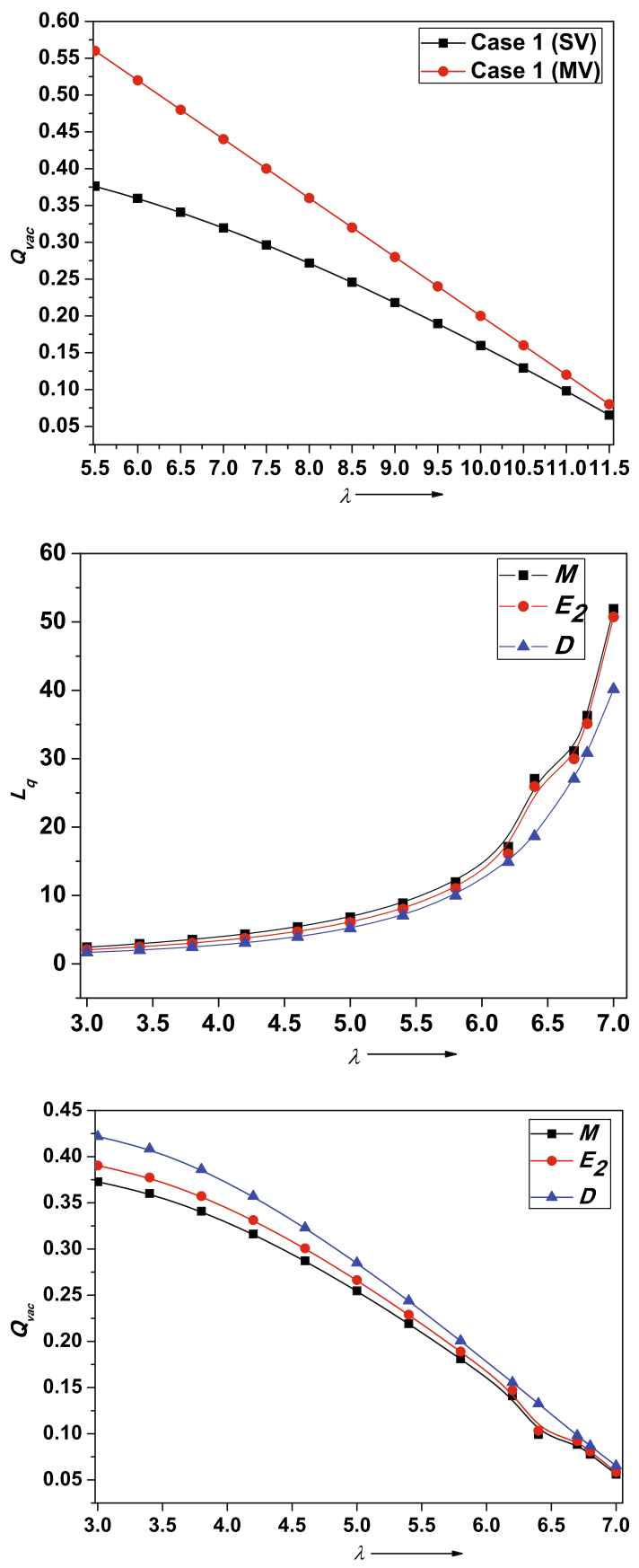
achieved or not. Actually, we can conclude from the explanation presented above that our model helps reducing congestion in the real-life queues because of QSDV.

\section{Cost Model}

In this section, we present a cost model which may help in deciding the optimal values of the system parameters to minimize the total system cost. This type of cost model may be applicable in the example of group testing (viz., for sample of testing COVID-19) that has been discussed in introduction section. For this purpose, we consider the following cost parameters:

$C_{s} \equiv$ Startup cost (i.e., cost that bring the sample to the system) per sample per unit time.

$C_{b} \equiv$ Holding cost (i.e., cost to preserve the sample waiting in the queue for test, during health worker's busy period) per sample per unit time.

$C_{v} \equiv$ Holding cost (i.e., cost to preserve the sample waiting in the queue for test, during health worker's vacation period) per sample per unit time.

$C_{d} \equiv$ Holding cost (i.e., cost to preserve the sample waiting in the queue for test, during health worker's dormant period) per sample per unit time (exists only for SV).

$C_{o} \equiv$ Testing cost (i.e., cost when sample is taken for testing by health worker) per sample per unit time. Thus in long run,

total system cost $(\mathrm{TSC})=\lambda C_{s}+C_{b} \sum_{n=0}^{\infty} \sum_{r=a}^{b} n \frac{\alpha_{n, r}}{P_{\text {busy }}}+C_{v} \sum_{n=0}^{\infty} \sum_{k=0}^{\min (n, a-1)} n \frac{\beta_{n}^{[k]}}{Q_{\text {vac }}}+$ $(1-\delta) C_{d} \sum_{n=0}^{a-1} n \frac{f_{n}}{P_{d o r}}+C_{o} L^{s e r}$.

Here, we present a numerical result by considering particular values of the system parameters. We vary the value of $a$ from 1 to 12 and fix the value of $b$ at 12 . In Table 1 we present the values of TSC for MV (see column 2-5 of Table 1) and for SV (see column 6-9 of Table 1) and for different values of $\lambda=7,10,13,18$. The service time distribution is considered to be $E_{2}$ with batch size dependent service rate $\mu_{r}=r \mu(\mu=0.15)$ and the vacation time distribution is considered to be exponential distribution with queue size dependent vacation rate $v_{k}=v_{k-1}+1\left(1 \leq k \leq a-1, v_{0}=1.5\right)$. The TSC are obtained under the consideration $C_{s}=0.1, C_{b}=1.0, C_{v}=1.5, C_{d}=1.7$, and $C_{o}=3.5$.

By considering this type of numerical experiment with desired values of system parameters and service time distribution (vacation time distribution) system analysts may easily achieve the minimum TSC by considering an optimal value of $a$. For fixed $\lambda$ the minimum values of TSC are indicated in bold letters and the corresponding values of $a$ are the desired optimum values of the lower threshold. For example for $\lambda=10$ (MV), the minimum TSC is 46.8892 which is achieved at $a=4$, hence, $a=4$ is the corresponding optimum value. A similar conclusion may be drawn for all other values of $\lambda$ and for SV and MV.

\section{Conclusion}

In this article, we dealt with an infinite capacity batch service queue with single and multiple vacations where the service time of the batches depend on the size of the batch under service, and the vacation time of the server depends on the queue size at vacation initiation epoch. Steady-state joint probabilities have been achieved at various epochs by using the supplementary variable approach and the bivariate generating function method. Finally, various 


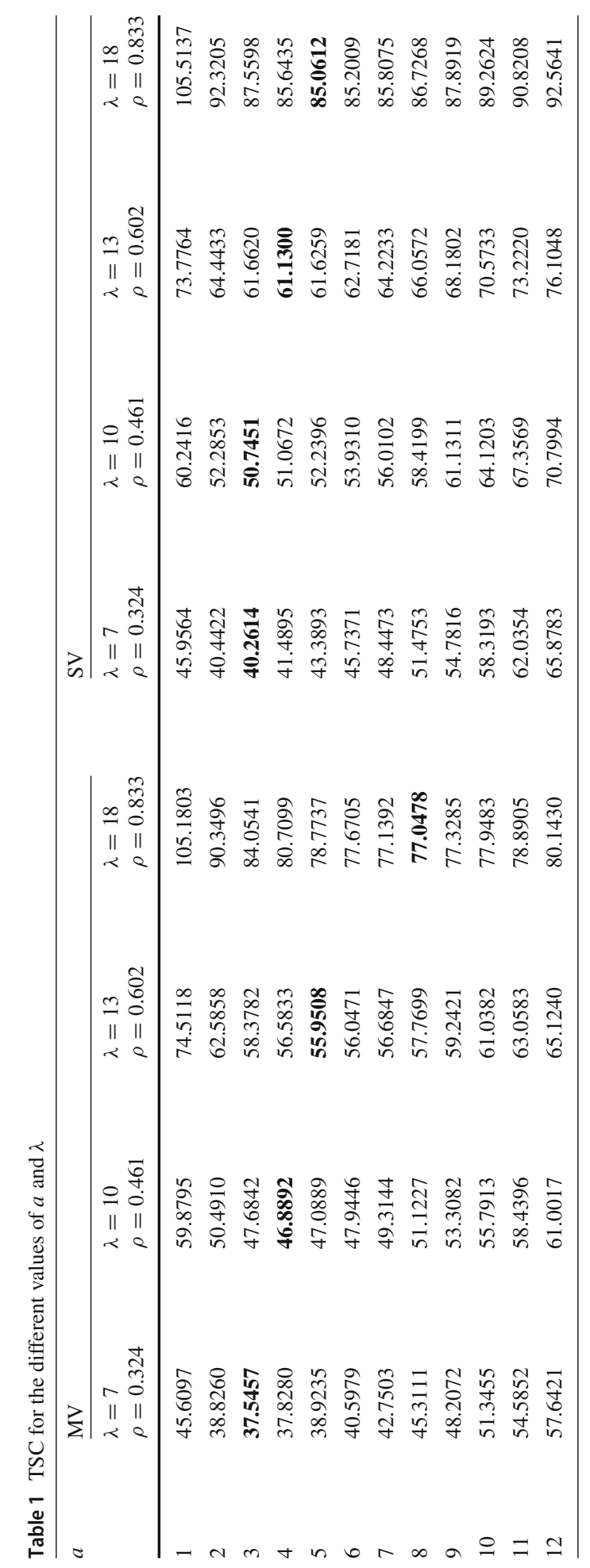


performance measures have been discussed to appraise the applicability of the considered model in the numerical section. In the present model interarrival time has been considered to be exponentially distributed, however, in computer communication and telecommunication system the arrivals of the data are bursty in nature and hence cannot be model using exponential interarrival time. To model these type of real-life system, the analysis of the present model may motivate researchers to analyze a more complex model for the steady-state joint probabilities for different service rules (different vacation policies) with more general arrival (service) process, viz., $M A P(M S P)$ or $B M A P(B M S P)$.

Author Contributions Mr. GKT analyzed the model mathematically. Dr. AB was a major contributor in programming and writing the manuscript.

Funding This work was supported by the Council of Scientific \& Industrial Research (CSIR), India. (09/1217(0026)/2017-EMR-I)

\section{Declarations}

Conflict of interest The authors declare that they have no conflict of interest.

Data availability: Not applicable.

\section{References}

1. Gupta, U.C., Pradhan, S.: Queue length and server content distribution in an infinite-buffer batch-service queue with batch-size-dependent service. Adv. Opera. Res. 2015, 1-12 (2015)

2. Pradhan, S., Gupta, U.C., Samanta, S.K.: Analyzing an infinite buffer batch arrival and batch service queue under batch-size-dependent service policy. J. Korean Stat. Soc. 45(1), 137-148 (2016)

3. Bank, B., Samanta, S.K.: Analytical and computational studies of the $B M A P / G^{(a, Y)} / 1$ queue. Commun. Stat. Theor. Methods 50(15), 3586-3614, (2020). https://doi.org/10.1080/03610926.2019.1708941

4. Bar-Lev, S.K., Parlar, M., Perry, D., Stadje, W., Van der Duyn Schouten, F.A.: Applications of bulk queues to group testing models with incomplete identification. Eur. J. Oper. Res. 183(1), 226-237 (2007)

5. Chaudhry, M.L., Templeton, J.G.: First Course in Bulk Queues. A Wiley-interscience publication, Hoboken (1983)

6. Choi, B.D., Han, D.H.: $G / M^{(a, b)} / 1$ queues with server vacations. J. Oper. Res. Soc. Jpn. 37(3), 171-181 (1994)

7. Chow, W.K., Chow, C.L.: A discussion on implementing pooling detection tests of novel coronavirus (SARS-CoV-2) for a large population. Epidemiol. Infect. 149(e17), 1-6 (2021). https://doi.org/10.1017/ S0950268820003155

8. Doshi, B.T.: Queueing systems with vacations: a survey. Queueing Syst. 1(1), 29-66 (1986)

9. Frey, A., Takahashi, Y.: An explicit solution for an $M / G I / 1 / N$ queue with vacation time and exhaustive service discipline. J. Oper. Res. Soc. Jpn. 41(3), 430-441 (1998)

10. Gupta, G.K., Banerjee, A.: On finite buffer bulk arrival bulk service queue with queue length and batch size dependent service. Int. J. Appl. Comput. Math. 5(2), 32 (2019)

11. Gupta, G.K., Banerjee, A., Gupta, U.C.: On finite-buffer batch-size-dependent bulk service queue with queue-length dependent vacation. Qual. Technol. Quant. Manag. 17(5), 501-527 (2020). https://doi.org/ 10.1080/16843703.2019.1675568

12. Gupta, U.C., Sikdar, K.: The finite-buffer $M / G / 1$ queue with general bulk-service rule and single vacation. Perform. Eval. 57(2), 199-219 (2004)

13. Haridass, M., Arumuganathan, R.: Analysis of a $M^{X} / G(a, b) / 1$ queueing system with vacation interruption. RAIRO Oper. Res. 46(4), 305-334 (2012)

14. Jeyakumar, S., Senthilnathan, B.: Modelling and analysis of a $M^{X} / G^{(a, b)} / 1$ queue with multiple vacations, setup time, closedown time and server breakdown without interruption. Int. J. Oper. Res. 19(1), 114-139 (2014) 
15. Kalita, C.R., Choudhury, G.: Analysis of an unreliable $M^{X} / G 1 G 2 / 1$ repeated service queue with delayed repair under randomized vacation policy. Commun. Stat. Theory Methods 48(21), 5336-5369 (2019)

16. Karpagam, S., Ayyappan, G., Somasundaram, B.: A bulk queueing system with rework in manufacturing industry with starting failure and single vacation. Int. J. Appl. Comput. Math. 6(6), 1-22 (2020)

17. Ke, J.C., Wu, C.H., Zhang, Z.G.: Recent developments in vacation queueing models: a short survey. Int. J. Oper. Res. 7(4), 3-8 (2010)

18. Lee, H.W., Lee, S.S., Chae, K.C.: A fixed-size batch service queue with vacations. Int. J. Stoch. Anal. 9(2), 205-219 (1996)

19. Lee, H.W., Lee, S.S., Chae, K.C., Nadarajan, R.: On a batch service queue with single vacation. Appl. Math. Model. 16(1), 36-42 (1992)

20. Levy, Y., Yechiali, U.: Utilization of idle time in an $M / G / 1$ queueing system. Manag. Sci. 22(2), 202-211 (1975)

21. Medhi, J.: Stochastic Models in Queueing Theory. Academic Press, Cambridge (2002)

22. Neuts, M.F.: A general class of bulk queues with Poisson input. Ann. Math. Stat. 38(3), 759-770 (1967)

23. Pool testing of SARS-CoV-02 samples increases worldwide test capacities many times over. Retrieved from https://aktuelles.uni-frankfurt.de/englisch/pool-testing-of-sars-cov-02-samplesincreases-worldwide-test-capacities-many-times-over/. (2020)

24. Pradhan, S., Gupta, U.C.: Stationary queue and server content distribution of a batch-size-dependent service queue with batch Markovian arrival process: $B M A P / G_{n}^{(a, b)} / 1$. Commun. Stat. Theory Methods (2020). https://doi.org/10.1080/03610926.2020.1813304

25. Samanta, S.K., Chaudhry, M.L., Gupta, U.C.: Discrete-time $G e o^{X} / G^{(a, b)} / 1 / N$ queues with single and multiple vacations. Math. Comput. Model. 45(1-2), 93-108 (2007)

26. Sikdar, K., Gupta, U.C.: Analytic and numerical aspects of batch service queues with single vacation. Comput. Oper. Res. 32(4), 943-966 (2005)

27. Sikdar, K., Gupta, U.C.: On the batch arrival batch service queue with finite buffer under servers vacation: $M^{X} / G^{Y} / 1 / N$ queue. Comput. Math. Appl. 56(11), 2861-2873 (2008)

28. Sikdar, K., Samanta, S.K.: Analysis of a finite buffer variable batch service queue with batch Markovian arrival process and servers vacation. OPSEARCH 53(3), 553-583 (2016)

29. Takagi, H.: Queueing analysis: A foundation of performance evaluation. Vol. 1, Vacation and Priority, North-Holland, New York. (1991)

30. Tamrakar, G.K., Banerjee, A.: On steady-state joint distribution of an infinite buffer batch service Poisson queue with single and multiple vacation. OPSEARCH, 57(4), 1337-1373 (2020). https://doi.org/10.1007/ s12597-020-00446-9

31. Tian, N., Zhang, Z.G.: Vacation Queueing Models: Theory and Applications, vol. 93. Springer Science \& Business Media, New York (2006)

32. Vadivu, A.S., Arumuganathan, R.: Cost analysis of $M A P / G^{(a, b)} / 1 / N$ queue with multiple vacations and closedown times. Qual. Technol. Quant. Manag. 12(4), 605-626 (2015)

33. Yelin, I., Aharony, N., Shaer-Tamar, E., Argoetti, A., Messer, E., Berenbaum, D., et al.: Evaluation of Covid-19 RT-qPCR test in multi sample pools. Clin. Infect. Dis. 71(16), 2073-2078 (2020)

Publisher's Note Springer Nature remains neutral with regard to jurisdictional claims in published maps and institutional affiliations. 